\title{
Evaluation of the properties of plastic-metal interfaces directly bonded via injection molding
}

\author{
Shin Horiuchi ${ }^{1,}$, Nao Terasaki ${ }^{1,2}$, and Masami Itabashi ${ }^{3}$ \\ ${ }^{1}$ Research Laboratory for Adhesion and Interfacial Phenomena, AIST, 1-1-1, Higashi, Tsukuba, Ibaraki 305-8565, Japan \\ 2 Advanced Manufacturing Research Institute, National Institute of Advanced Industrial Science and Technology (AIST), \\ 807-1 Shuku-machi, Tosu, Saga 841-0052, Japan \\ 3 Taisei Plas, Co., Ltd., 10-5-1, Honcho, Nihonbashi, Chuo-ku, Tokyo 103-0023, Japan
}

Received: 12 November 2019 / Accepted: 15 January 2020

\begin{abstract}
Plastic and metal can be directly bonded via insert injection molding by creating surface porous structures with 30-50 nm sizes on metal surface. Molten polymers can be penetrated into the small pores created on metal surface in the short molding process, and strong joint can be obtained. The bonding mechanism of aluminum alloy (Al5052) and polyphenylene sulfide (PPS) with two different surface features of Al5052 was studied. Scanning transmission electron microscopy (STEM) was performed to investigate the interfacial structures in terms of elemental distributions and three-dimensional (3D) inter-connectivity of the pores on the Al5052, and the chemical reaction which takes place during the joint process was investigated by X-ray photon spectroscopy (XPS). The properties of the joint interfaces were evaluated to discuss the effect of interfacial structures on the joint properties.
\end{abstract}

Keywords: Plastic / metal / bonding mechanism / fracture / electron microscopy

\section{Introduction}

Dissimilar joining of hard (metal) and soft (plastic) materials is one of the key technologies for the production of lightweight and energy-efficient vehicles and aircrafts, which have been desired for the saving of energy consumption and global carbon dioxide emission. The application of adhesively bonded joints has increased significantly in recent years to join dissimilar materials and has been growingly used in automotive industry [1]. In this work, a new metal/plastic joint technology is reported, which enables to assemble metal and plastic directly without adhesive. In this process, surface of metallic parts is chemically etched to produce extremely small pores on the surface. Such surface-modified metals are inserted into a mold and plastic can be directly bonded to metal by injection molding, which is called as nano molding technology (NMT) [2,3]. Metal-plastic hybrid structures with various metal/plastic combinations, such as $\mathrm{Al}, \mathrm{Cu}, \mathrm{Ti}$, $\mathrm{Mg}$ as metals and PPS (polyphenylene sulfide), PA (polyamide) and PP (polypropylene) as plastics, can be obtained. ${ }^{1}$ Remarkable strong metal-plastic joint strength can be obtained through the penetration of the polymer chains into the nano-sized pores on the metal. One of the benefits of such direct joint technique is the capability to

\footnotetext{
* e-mail: s.horiuchi@aist.go.jp

${ }^{1}$ https://taiseiplas.jp/nmt/
}

assemble metal and plastic in a short time with high design flexibility once the molding condition is determined. Although the size of the products manufactured via injection molding is restricted mainly by a mold size, cost reduction could be achieved when a large number of products are repeatedly manufactured. Adhesive bonding, on the other hand, has an advantage on multimaterial joining with all types of materials and sizes of products are not as limited as the injection molding process. Adequate understanding of the joint mechanism and appropriate evaluation methodology are necessary to ensure safety and reliability of the new joint technology. In this work, the mechanism of the joint process of PPS and Al5052 is investigated by electron microscopy and XPS. New test methods to evaluate the joint performance including assessment of the durability under various environmental conditions are also developed and the mechanism behind the high bonding strength and its failure is discussed.

\section{Experimental}

\subsection{Sample preparation}

The test specimens for the evaluation of the joint strength were prepared by insert-injection molding of PPS (SGX120, TOSO Corp., Japan) onto surface-modified Al5052 plate at the melt temperatures of $290-330^{\circ} \mathrm{C}$ and the mold 
temperature of $120^{\circ} \mathrm{C}$. Two types of surface structures of Al5052 are prepared by the chemical treatment developed by Taisei Plas Co., Ltd. using a hydrazine-based aqueous solution [3]. Those two are different in terms of the pore size, the depth of the treated layer and the 3D interconnected structures inside of the surface layers. The PPS/ Al5052 joint performances were evaluated in accordance with ISO19095 [4]. The tensile joint strength was evaluated with the butt-joint specimen where PPS and Al parts are simply jointed at the ends of their pieces with $10 \mathrm{~mm}$ width, $50 \mathrm{~mm}$ length, and $2 \mathrm{~mm}$ thickness. The lap shear strength was evaluated with the lap joint specimen where Al plate with $50 \mathrm{~mm}$ length, $18 \mathrm{~mm}$ width and $1.5 \mathrm{~mm}$ thickness and PPS with $50 \mathrm{~mm}$ length, $10 \mathrm{~mm}$ width and $3 \mathrm{~mm}$ thickness are jointed with the joint area of $5 \times 10 \mathrm{~mm}$. Peeling resistivity was measured by floating roller method with the joint specimen of PPS plate with $4 \mathrm{~mm}$ thickness and $\mathrm{Al}$ foil with $0.4 \mathrm{~mm}$ thickness. Accelerated durability tests under high humidity/temperature and rapid-rate thermal cycle conditions were carried out by using a constant climate cabinet (LH-113, Espec Corp., Japan) and rapid-rate thermal cycle chamber (TSE-12-A, Espec Corp., Japan), respectively. The fracture energy of the joint interfaces was also evaluated by the method described in detail later. Adhesive bonding of PPS and Al5052 with two-component epoxy adhesive (Denatite XNR2204/XNH3324, Nagase Chemtex Corp., Japan) cured at $100{ }^{\circ} \mathrm{C}$ for $30 \mathrm{~min}$ was also evaluated. All the tests were performed with 5 specimens, and average and standard deviation were calculated.

\subsection{Characterization of the joint interfaces}

The PPS/Al5052 interfacial structures were investigated by electron microscopy using a TECNAI Osiris (FEI company, USA) scanning transmission electron microscope (STEM) with an accelerating voltage of $200 \mathrm{kV}$. Energydispersive X-ray (EDX) microanalysis and STEM/EDX elemental mapping were performed with 4 windowless silicon drift EDX detectors (Super-XTM), which were placed symmetrically around the optical axis near the specimen area [5]. STEM-tomography was performed to construct detailed 3D structures of the joint interface [6], where series of STEM images by sequentially tilting a specimen in the tilt range of \pm 60 degrees. $3 \mathrm{D}$ images were reconstructed with the collected tilt-series using Avizo software (Avizo 2019.2, Thermo Fisher Scientific Inc., Lyon). Thin specimens with the thickness of about $70 \mathrm{~nm}$ were prepared by cutting using ultramicrotomy; the cutting direction was adjusted to an angle of $90^{\circ}$ between the interface and the edge of the diamond knife. The fracture surfaces after the fracture resistivity measurements were investigated by scanning electron microscopy with Carl Zeiss Ultra 55 at an accelerating voltage of $2 \mathrm{kV}$.

Chemical structures of Al5052 at the interfaces were investigated by XPS depth profiling using PHI5000 Versal Probe instrument with a $\mathrm{Mg} \mathrm{K} \alpha \mathrm{X}$-ray source. For sputter depth profiling, $\mathrm{Ar}^{+}$ions of $2 \mathrm{keV}$ energy was used [7].

As a mechanoluminescent (ML) sensor, green-emitting europium-doped strontium aluminate $\left(\mathrm{SrAl}_{2} \mathrm{O}_{4}: \mathrm{Eu}^{2+}\right.$, denoted as SAOE, $\lambda_{\text {em }}=520 \mathrm{~nm}$ ) was used to visualize the crack propagation during the fracture of the joint interfaces. Here, a commercial ML paint (ML-32ET, Sakai Chemical. Japan), consisting of the SAOE ML material and epoxy resin, was sprayed onto the specimens to obtain ML films (thickness: approximately $50 \mu \mathrm{m}$ ) [8,9]. For quantitative and reproductive $\mathrm{ML}$ behavior, a tensile load was applied to the specimen after irradiation using blue light ( $\lambda=470 \mathrm{~nm}, 1 \mathrm{~min}$ ) for excitation. The mechanoluminescence was recorded using high speed CCD cameras (FASTCAM Mini AX, PHOTRON Ltd., Tokyo) in 30000 frame/s (fps) accompanied by a speed crack propagation on the CT specimen.

\section{Results and discussion}

\subsection{Bonding mechanism}

The bonding mechanism is studied by using two types of Al5052 surface structures, which are called as NMT1 and NMT2, differing in pore size and shape, the thickness of the modified layers and internal pore connectivity. Figure 1a and b shows SEM micrographs representing the top views of the Al5052 surface features of NMT1 and NMT2 before bonding, respectively. In the both treatments, quite small pores are produced on the $\mathrm{Al}$ surface with about $20 \mathrm{~nm}$ diameter. The pores of NMT1 are relatively smaller than those of NMT2 and the shapes of the pores of NMT1 seems to be irregular, while those of NMT2 are uniformly distributed with the micrometer-scale slight roughness.

Figure 2 shows the cross-sectional views of the PPS/ Al5052 joint interface of NMT1 by a STEM high angle annular dark field (HAADF) image (Fig. 2a) and STEMEDX elemental maps of $\mathrm{Al}$, sulfur $(\mathrm{S})$ and oxygen $(\mathrm{O})$ (Fig. 2b-d, respectively). The HAADF image shows that the depth of the surface modified layer is approximately $100 \mathrm{~nm}$. The elemental maps indicate that the surface modified layer contains aluminum and oxygen, and that PPS, which is represented by the $\mathrm{S}$ map (Fig. 2c), can be penetrated into the Al surface pores without voids. NMT2, on the other hand, has thick interfacial layer with approximately $500 \mathrm{~nm}$ thickness as presented in Figure 3. The STEM bright field (BF) image shows that PPS is penetrated only into the upper part with about $100 \mathrm{~nm}$ depth, and the most part of the layer remains with empty holes. The S map (Fig. 2c) clearly indicates that the penetration of PPS into the pores is not completed.

Figure 4 shows the EDX spectra obtained from the interfacial region indicated in Figure 2a. It shows $\mathrm{S} \mathrm{K} \alpha$ peak at $2.35 \mathrm{keV}$, indicating that the pores are filled with PPS in the entire modified layer. Figure 5, on the other hand, shows the spectra acquired from the two regions indicated in Figure 3a. It is confirmed that the bottom part of the layer does not contain PPS where the most part of the layer remains with empty holes.

Electron tomography allows us to understand the details of the porous structures created on the Al surface. Figure 6 shows typical 3D views of (a) NMT1 and (b) NMT2. We can understand that the pores are not isolated but rather are connected to each other forming 3D continuous hollows whose space is $10-20 \mathrm{~nm}$ in width in NMT1, while NMT2 shows the isolated pores grown perpendicular from the substrate. It is indicated that the 

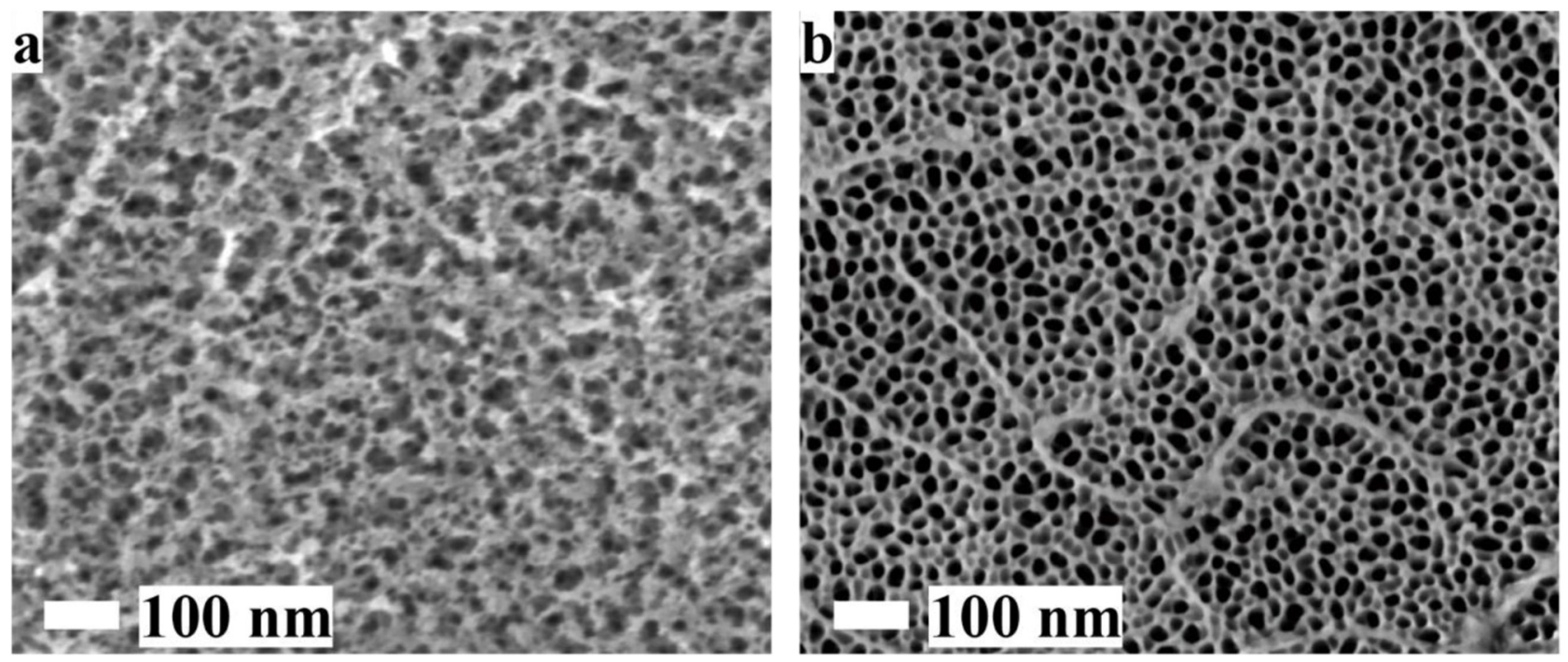

Fig. 1. SEM micrographs showing the surface porous features of (a) NMT1 and (b) NMT2.
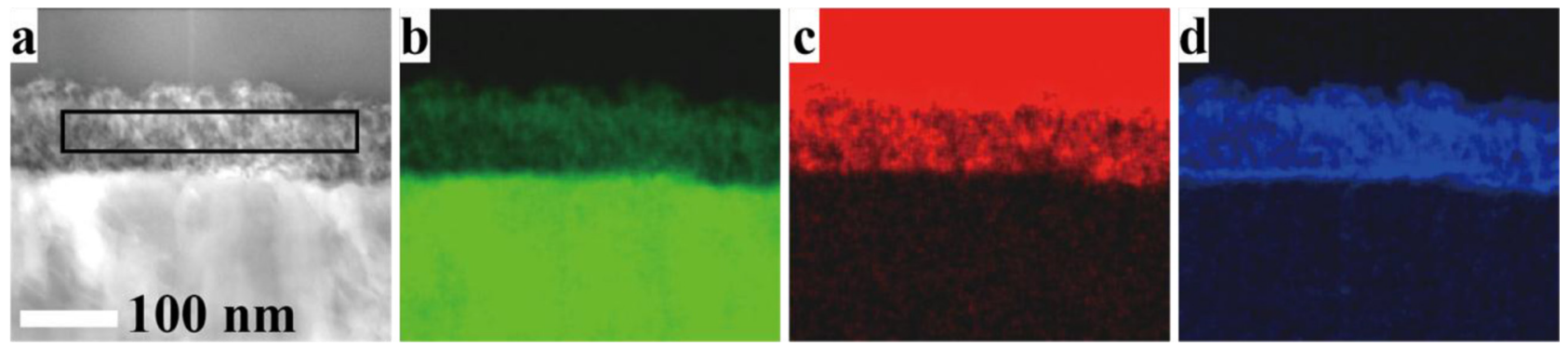

Fig. 2. Cross-sectional views of PPS/A15052 joint interfacial region with NMT1 surface treatment. (a) STEM-HAADF image and (b)-(d) EDX elemental maps of $\mathrm{Al}, \mathrm{S}$ and $\mathrm{O}$, respectively.
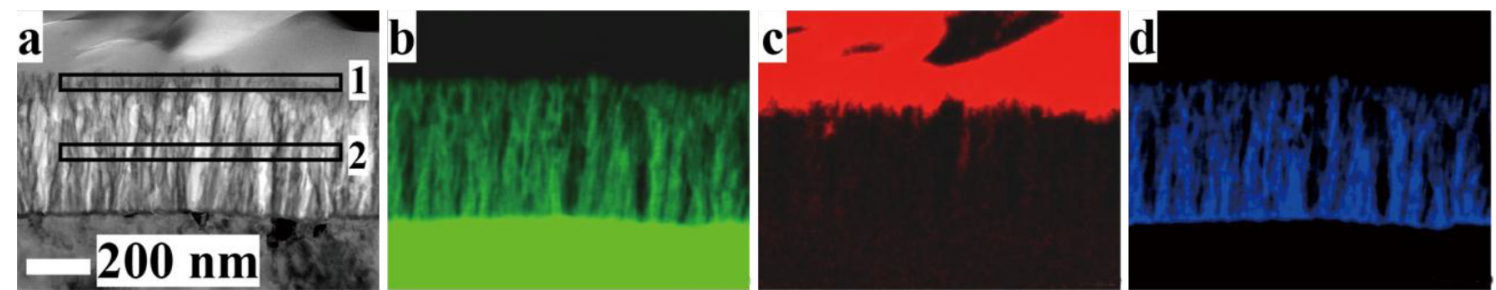

Fig. 3. Cross-sectional views of PPS/5052 joint interface with NMT2 surface treatment. (a) STEM-BF image and (b)-(d) EDX elemental maps of $\mathrm{Al}, \mathrm{S}$ and $\mathrm{O}$, respectively.

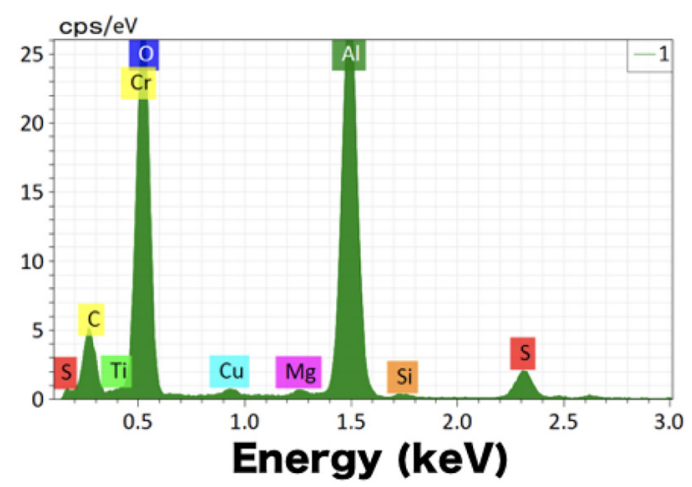

Fig. 4. EDX spectrum acquired from the interfacial region of the joint with NMT1 surface treatment indicated as the region in Figure 2a. polymer chains can go into the complicated narrow and deep hollows during the injection molding process within a few minutes in NMT1. Due to the continuous porous structure in NMT1, air can escape from the pores and then the polymer chains are replaced with it. On the other hand, NMT2 gives the one way narrow space and thus the PPS polymer chains cannot push out air from the pores, resulting in incomplete penetration for polymer chains into the upper part of the layer.

Further investigation for metal/polymer chemical interaction by XPS depth profiles was employed. Figure $7 \mathrm{a}$ shows the XPS depth profiles of $\mathrm{Al} 2 p$ peaks obtained from NMT1 before the bonding. The peak was gradually shifted toward higher binding energy within the initial $1 \mathrm{~min}$, then another peak appeared and finally the single peak corresponding to the metallic $\mathrm{Al}(73 \mathrm{eV})$ 

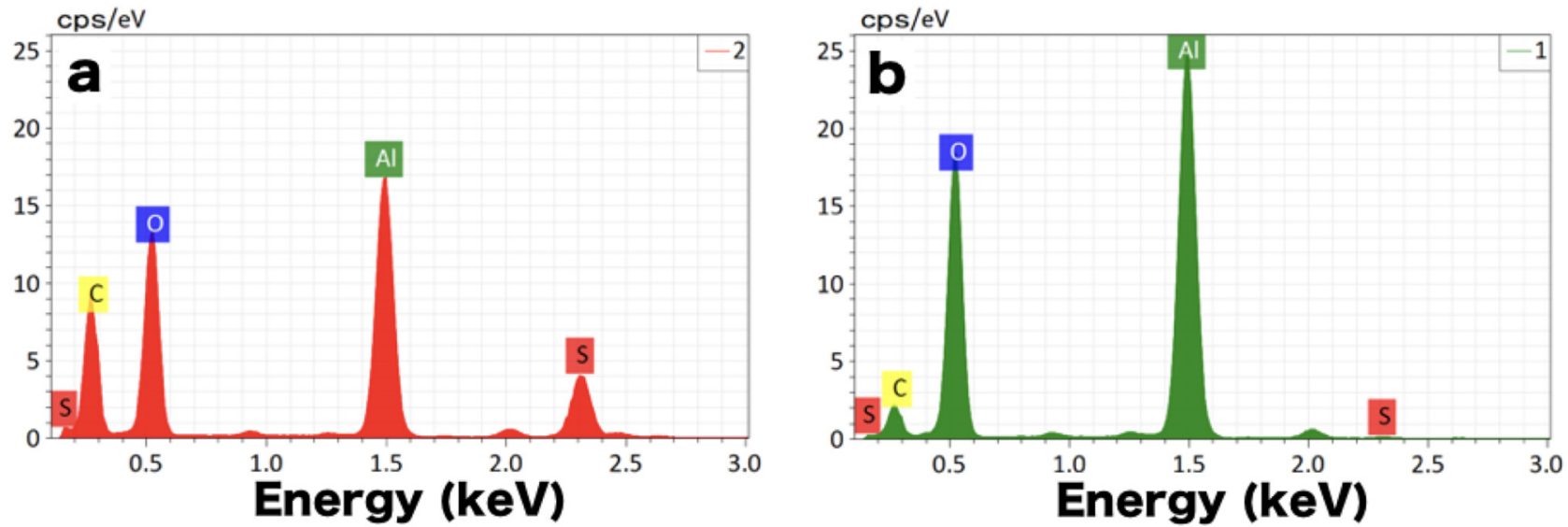

Fig. 5. EDX spectra acquired from the upper (a) and bottom (b) parts of the interfacial region of the joint with NMT2 surface treatment indicated as the regions in Figure 3a.
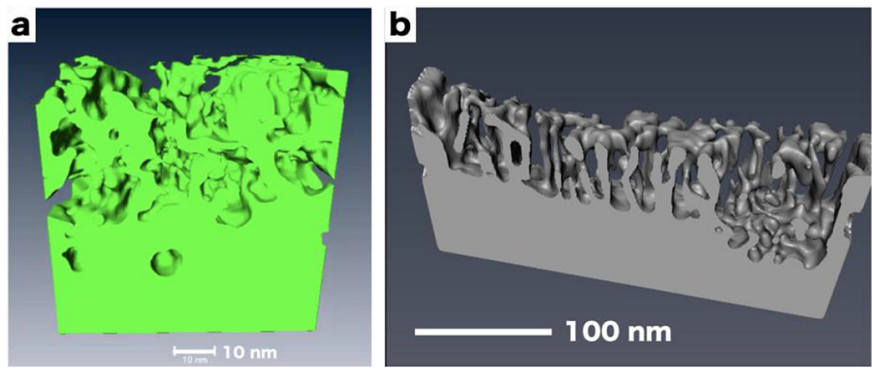

Fig. 6. 3D re-constructed views of (a) NMT1 and (b) NMT2 showing the internal porous structures created on the Al surface.

remains after the $11 \mathrm{~min}$ of the sputtering. The depth profiles clearly indicate that the chemical state of $\mathrm{Al}$ in the oxygen-containing $\mathrm{Al}$ modified layer changes from the outermost surface to the inside. This means that the modified layer has a chemical gradient structure. XPS depth profiles for the PPS/Al5052 interface were obtained after removing the $\mathrm{Al}$ part by $\mathrm{HCl}$ etching as shown in Figure 7b. Even after the $\mathrm{HCl}$ etching, a trace of the $\mathrm{Al}$ remains on the $\mathrm{PPS}$, and $\mathrm{Al} 2 p$ peaks were able to be detected during the $\mathrm{Ar}^{+}$sputtering. The binding energies at which the $\mathrm{Al} 2 p$ peaks appeared were almost constant independent of the sputtering time and differed from those obtained before the bonding. It suggests that the bonding of PPS to the Al induces the changes in the chemical structure of the oxygen-containing Al surface layer.

Figure 8 shows the changes in the $\mathrm{O} / \mathrm{Al}$ atomic ratios calculated using the integral peak intensities of $\mathrm{Al} 2 p$ and $\mathrm{O}$ $1 s$ peaks as a function of the sputtering time obtained from profiles before the bonding and of the $\mathrm{Al} / \mathrm{PPS}$ interface. It indicates that the oxygen contains at higher ratios before the bonding in the entire depth of the modified layer. Especially, the outermost surface of the modified Al before the bonding has the highest $\mathrm{O} / \mathrm{Al}$ ratio, which is nearly equal to the value of $\mathrm{Al}(\mathrm{OH})_{3}$ and gradually decrease to approximately 2.0. Therefore, the chemical shift of the $\mathrm{Al}$ $2 p$ peaks observed before the bonding (Fig. $5 \mathrm{a}$ ) is caused by those gradual changes in the chemical structure from the
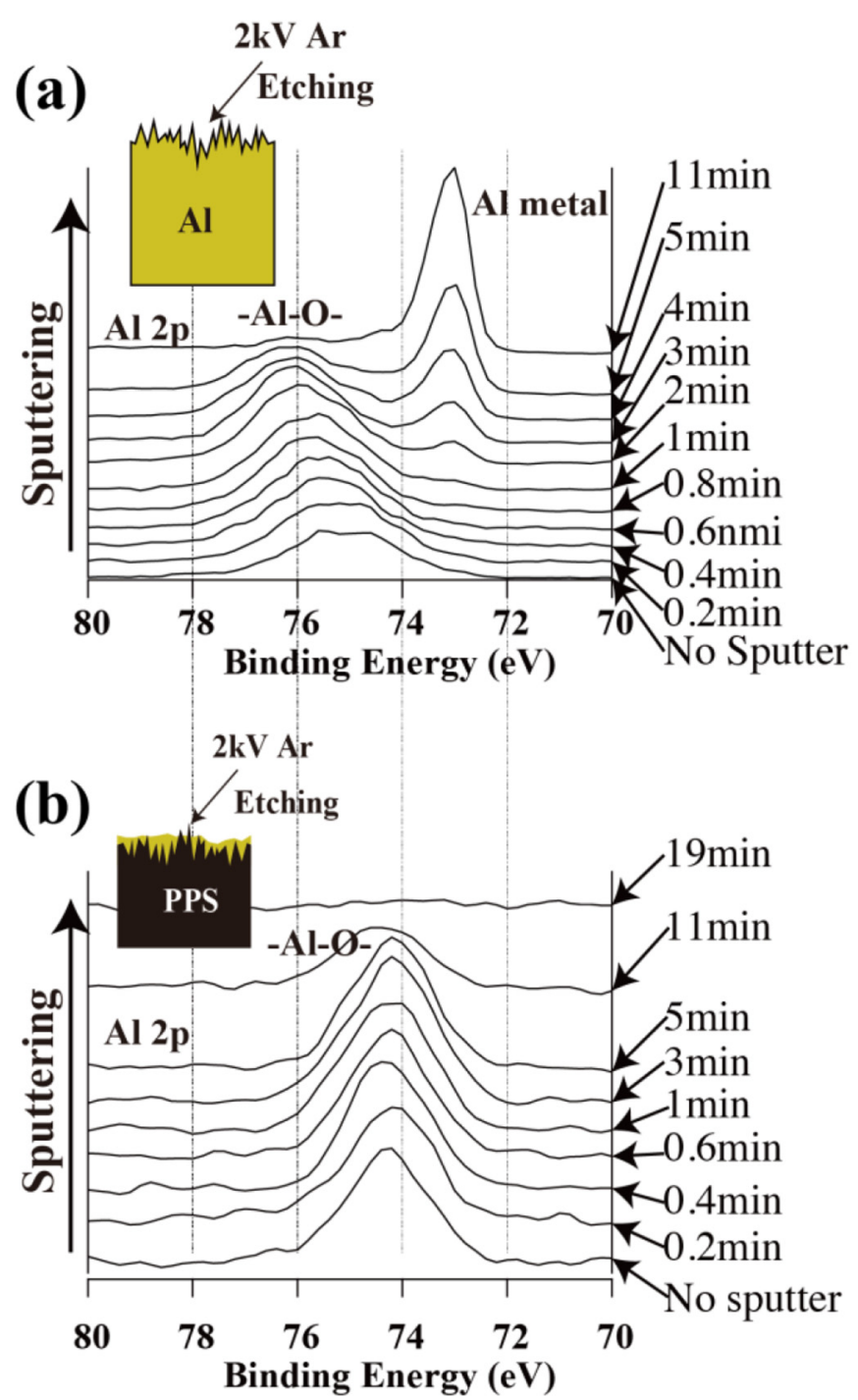

Fig. 7. XPS depth profiles of Al $2 p$ signals of (a) the Al surface before the bonding and of (b) the Al/PPS interface after the removal of $\mathrm{Al}$ by $\mathrm{HCl}$ etching. 
surface to the inner part of the $\mathrm{Al}$ modified layer. The dehydration of aluminum hydroxide in an air atmosphere has been reported to proceed according to the following reactions.

$$
\begin{gathered}
\mathrm{Al}(\mathrm{OH})_{3} \rightarrow \mathrm{AlO}(\mathrm{OH})+\mathrm{H}_{2} \mathrm{O} \\
2 \mathrm{AlOOH} \rightarrow \mathrm{Al}_{2} \mathrm{O}_{3}+\mathrm{H}_{2} \mathrm{O}
\end{gathered}
$$

$\mathrm{Al}(\mathrm{OH})_{3}$ is dehydrated to $\mathrm{AlO}(\mathrm{OH})$ in elevated temperature over $253^{\circ} \mathrm{C}$ in the first, and then $\mathrm{AlO}(\mathrm{OH})$ is dehydrated to $\mathrm{Al}_{2} \mathrm{O}_{3}$ over $500^{\circ} \mathrm{C}$. These reactions are endothermic with the activation energy of 15.70 and

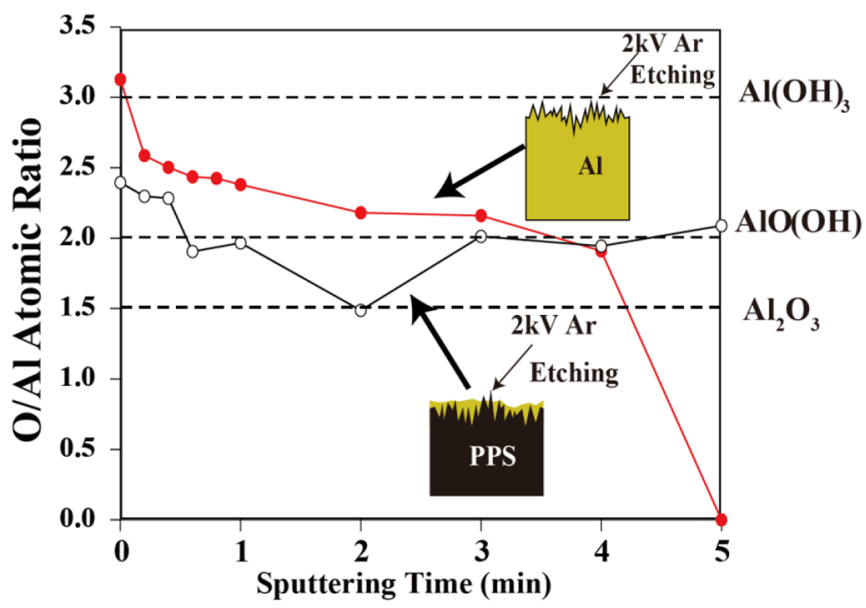

Fig. 8. $\mathrm{O} / \mathrm{Al}$ atomic ratios calculated using the $\mathrm{Al} 2 p$ and the $\mathrm{O}$ $1 s$ integral peak intensities as a function of sputtering time before (Al surface) and after (PPS/Al5052 interface) the bonding.
$0.20 \mathrm{~kJ} / \mathrm{mole}$, respectively $[10,11]$. When the hot molten polymer is contacted to the $\mathrm{Al}$ surface, these dehydration reactions take place with absorption of heat of the polymer. This heat transfer is assumed to promote the spontaneous penetration of the polymer chains into the pores.

The space provided by the pores is approximately equal to the one occupied with a few polymer chains. It could be thus difficult for high-viscous polymer melt to be penetrated into such extremely small pores. It is assumed that the 3D continuous hollow structure plays a critical role in the formation of the metal/polymer interface, which can help air escape from the pores when the polymer chains are penetrated into the pores.

\subsection{Evaluation of Al/PPS joint properties}

Figure 9 shows the durability of the PPS / Al5052 joint with NMT1 surface treatment under high humidity and rapidrate temperature changes. Figure 9a shows the tensile buttjoint strength under the humidity condition at $85^{\circ} \mathrm{C}$ and $85 \% \mathrm{RH}$, where the butt-joint specimens before and after the test are shown. The specimens were fractured near the metal/plastic interface and the initial tensile strength measured to be about $45 \mathrm{MPa}$ was maintained for $3000 \mathrm{~h}$. Since the tensile strength of the PPS is about $120 \mathrm{MPa}$, which is much higher than the measured joint strengths, the measured strengths represent the strengths of the interfacial region. Figure $9 \mathrm{~b}$ shows the thermal shock resistance under two conditions. The joint strength can be maintained even after the 1500 cycles of the rapid-rate temperature changes between -40 and $85^{\circ} \mathrm{C}$, while it decreases to about $25 \mathrm{MPa}$ with significant large scatterings of the strength between 500 and 1000 cycles of the larger temperature changes from -40 to $120^{\circ} \mathrm{C}$. Figure 10a
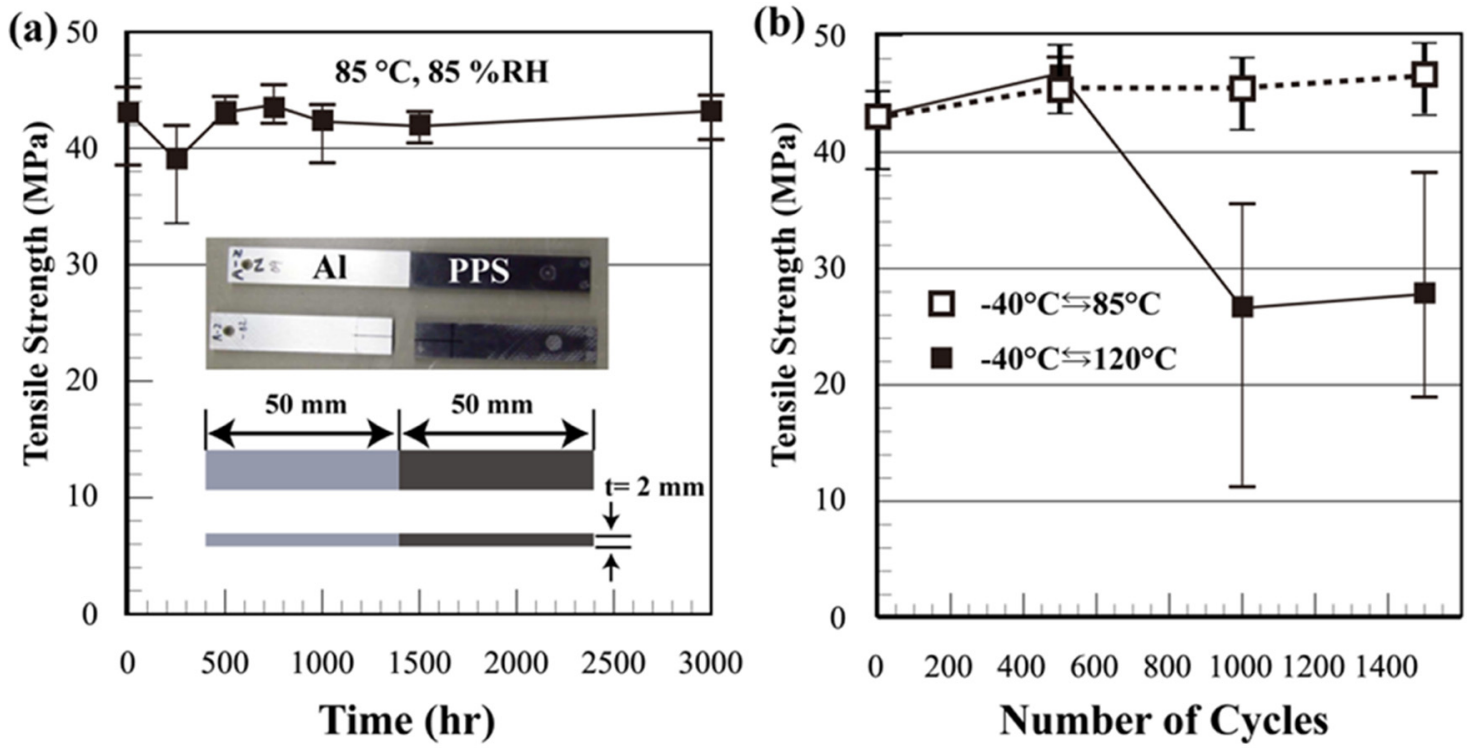

Fig. 9. Changes in the PPS/Al5052 tensile joint strength under accelerated environmental conditions. (a) High humidity and high temperature durability at $85^{\circ} \mathrm{C}$ and $85 \% \mathrm{RH}$. The butt-joint test specimens before and after the test are shown therein. (b) Thermal shock resistivity under the conditions from -40 to $85^{\circ} \mathrm{C}$ and from -40 to $120^{\circ} \mathrm{C}$. Each data is shown as the average of 5 tests with maximum and minimum results. 

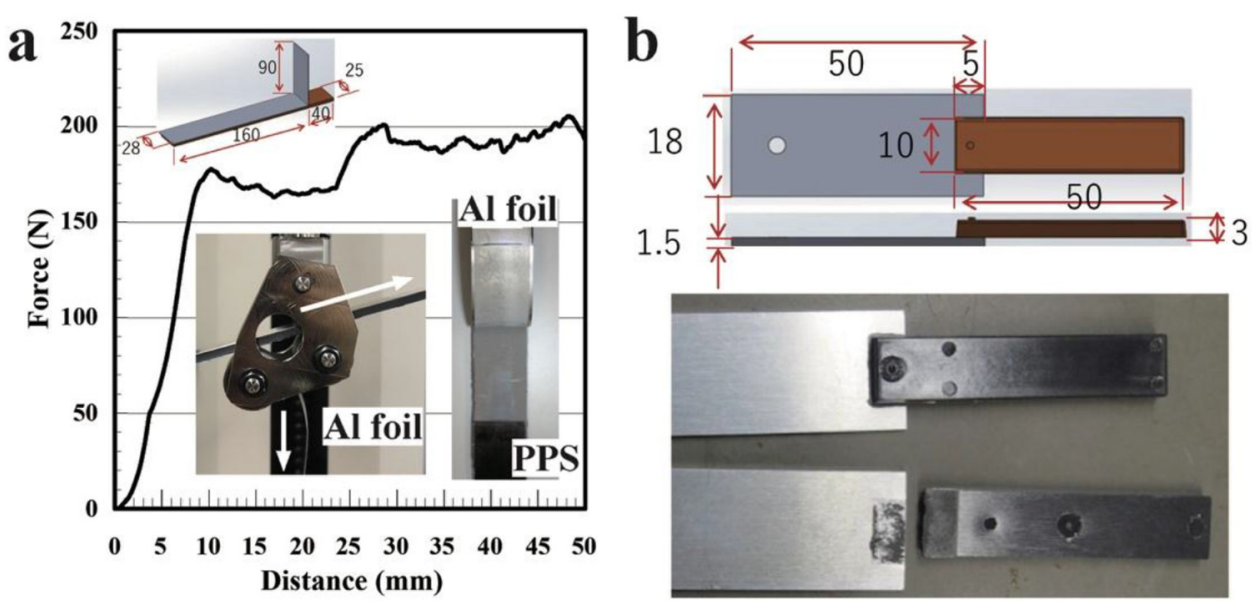

Fig. 10. (a) Force-distance curve obtained by the peeling resistivity test of Al/PPS laminate with NMT1 surface treatment. The specimen geometry, a specimen set in the floating roller jig, and the specimen after the test are shown therein. (b) The specimen geometry of the PPS/A15052 lap joint, and the test specimens before and after the test.
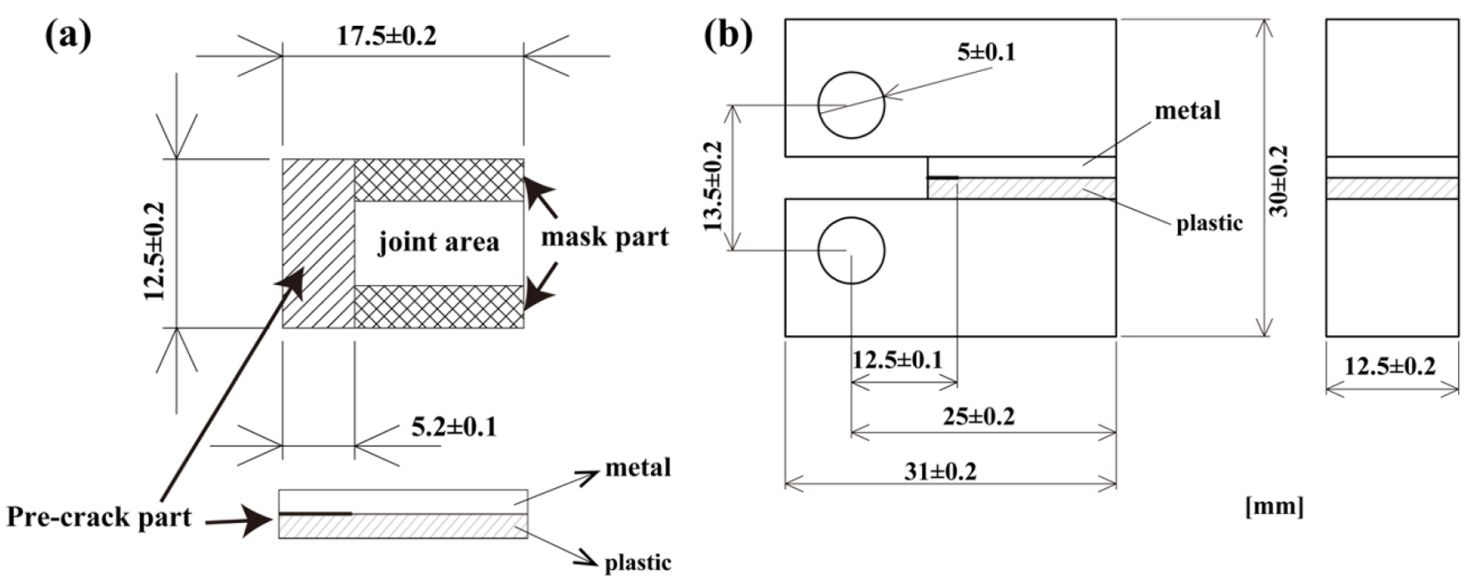

Fig. 11. Test specimen geometry and size for the measurement of interfacial fracture energy. (a) Metal/plastic laminate and (b) test specimen for the fracture test.

is a typical force-distance curve obtained in the peel resistance measurement by floating roller method, showing stable peeling with the force of about $180 \mathrm{~N} / 25 \mathrm{~mm}$. The specimen geometry for the peeling test, a specimen set in the floating roller jig and the specimen after the test are shown therein, showing that thin layer of PPS remains on the $\mathrm{Al}$ foil. Figure 10b shows the geometry of the lap joint specimen and the specimen before and after the lap shear testing, which was measured to be $44.2 \pm 1.2 \mathrm{MPa}$. The failure occurred near the interface where small amount of PPS is partly fixed on the Al plate. Small amount PPS is attached to the fracture surfaces of $\mathrm{Al}$ in all the measurements, suggesting that PPS forms weak layer [12] near the interface during the joint process. This is caused by the difference in the cooling rate of PPS in the interfacial region and in the bulk part. The molten PPS at the temperatures of $290-330^{\circ} \mathrm{C}$ in the injection molding machine is rapidly cooled when it is injected into the $\mathrm{Al}$ plate at $120^{\circ} \mathrm{C}$. Therefore, the crystallization behavior of PPS near the interface is different from that in the bulk part. It is reasonable to assume that the crystallinity of PPS near the interface is lower than the bulk part due to the rapid cooling. The PPS/Al5052 joint has outstanding joint performance under various test conditions except for the thermal shock with the significant large temperature difference. This is due to the large discrepancy in the thermal expansion coefficient between metal and plastic.

Evaluation of the fracture energy of the PPS/Al5052 interfaces were employed with the test specimen designed considering the compact tensile (CT) test specimen geometry as defined in ISO 13586 [13] for the measurements of fracture toughness of bulk plastics. PPS/Al5052 joint laminate were prepared as shown in Figure 10a, where metal and plastic plates with $2 \pm 0.1 \mathrm{~mm}$ thickness were jointed having non-bonded region at the one side of the joint laminate, which is served as a pre-crack part. To apply stress to the tip of the pre-crack, the laminate is sandwiched with two metal load blocks, which are made of aluminum alloy, with one-component epoxy adhesive (Scotch Weld, EW-2040, 3M, USA) in accordance with the geometry as specified in Figure 11b. The adhesive bonded load block/laminate interface could be cleaved 


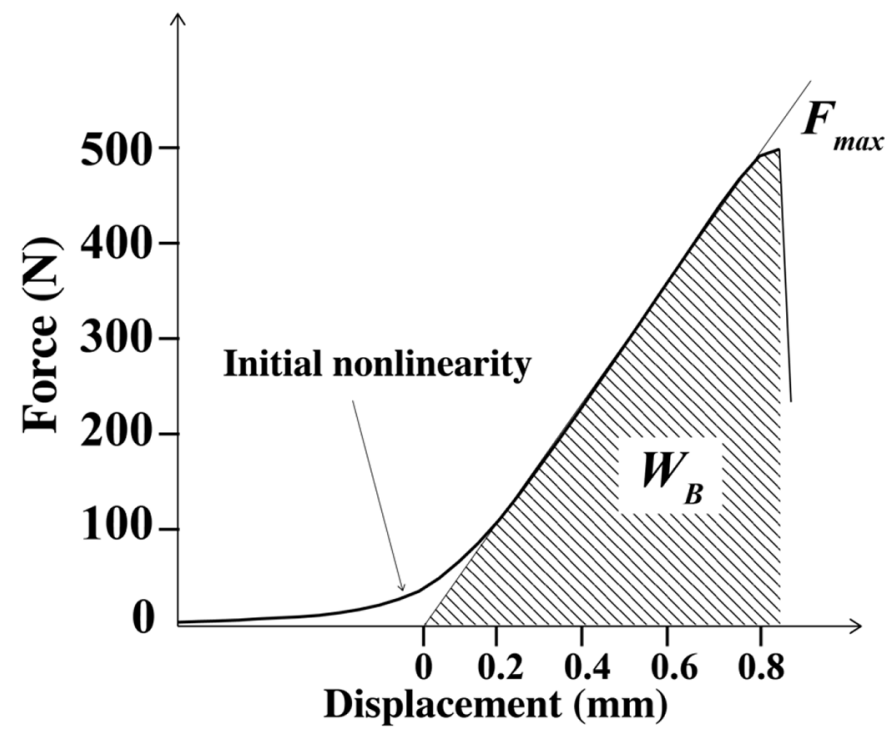

Fig. 12. Typical force-displacement curve obtained with $\mathrm{Al} / \mathrm{PPS}$ joint specimen and the definition of the energy $\left(W_{\mathrm{B}}\right)$ to be fractured.

before the fracture of the plastic-metal interface due to the high joint strength of the specimen. To avoid this problem, the joint area of the laminate is reduced to $50 \%$ as specified in Figure 11a by masking the surface of the substrate. Tensile force is applied to the specimen through the pins inserted into the two holes of the load blocks with the testing speed of $10 \mathrm{~mm} / \mathrm{min}$.

Figure 12 presents a typical force-displacement curve obtained with a PPS/Al5052 laminate with NMT1 surface treatment, which exhibits linear increment with an abrupt drop in the force at the instant of crack growth initiation. The energy to break $\left(W_{\mathrm{B}}\right)$ is calculated from the area under the force-displacement curve using the linear part of the curve. We performed an ML experiment with the aid of a high-speed camera to capture the fast phenomenon of the crack initiation and the growth during the test. Figure 13 shows sequential ML images during high speed crack propagation on the fracture test, taken using high-speed CCD camera (30 $000 \mathrm{fps})$. Until $8.18 \mathrm{~ms}$, ML luminance had been getting brighter and brighter at around the tip of the pre-crack, and then a ML point proceeded to the right direction through the bond line to be raptured within $0.08 \mathrm{~ms}(8.18-8.26 \mathrm{~ms})$. The ML point indicates strain concentration at crack tip and the following crack propagation. In addition, from the ML pattern appeared lower part, strain at the crack tip can be considered to be inclinedly generated in PPS lower part accompanied tensile load to the specimen. With the specimen having precracked part, the strongly bonded meta/plastic interfaces can be fractured along the interfaces, and the interfacial toughness can be quantitatively evaluated.

Table 1 summarizes the joint properties obtained in this work. It indicates that the NMT1 surface treatment gives slightly higher values as compared with the NMT2 in all the test conditions. The results of the adhesive bonding with NMT1 treatment were also shown, indicating that the

\section{Tip of pre-crack

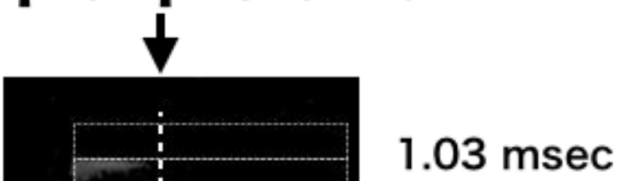

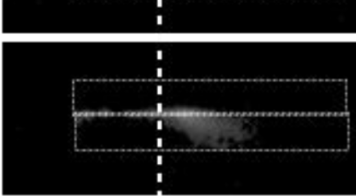

$8.18 \mathrm{msec}$

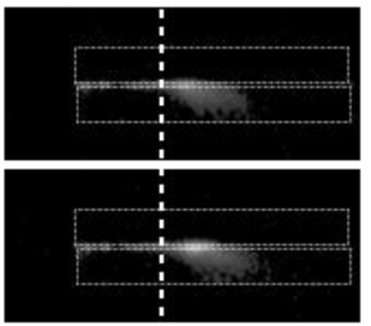

$8.19 \mathrm{msec}$

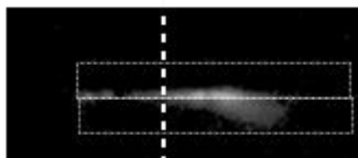

$8.20 \mathrm{msec}$

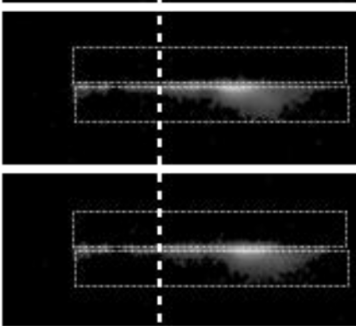

\section{$8.21 \mathrm{msec}$}

$8.22 \mathrm{msec}$

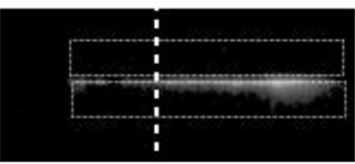

$8.23 \mathrm{msec}$

\section{$8.26 \mathrm{msec}$}

Fig. 13. ML experiments with high-speed camera to visualize the stress concentration at the crack tip. The upper part is $\mathrm{Al}$ and the lower part is PPS.

direct bonding gives extremely high joint strength than the adhesive bonding. Even though there is incomplete penetration of PPS into the surface pores in NMT2, it has sufficient joint strength. Figure 14 shows the fracture surfaces with different magnifications after the fracture energy measurements. The lowest magnification images (Fig. 14a and d) by optical microscopy indicate that the crack is initiated and is progressed along the PPS/Al interfaces near the pre-crack tips. The larger magnification images by SEM (Fig. 14b, c, e and f), however, indicate that PPS remains in almost all the areas in the both surface treatments. The naked Al surface with original surface pores are not found in the NMT1 as shown in Figure 14c, while it can be partially seen in the NMT2 as shown in Figure 14f. This means that the fracture occurs in mixed mode of the failure of the PPS part and the interfacial failure in NMT2 with pulling off the PPS polymer chains from the pores. 
Table 1. Summary of the joint properties of the PPS/Al5052 joints with NMT1 and NMT2 surface treatment.

\begin{tabular}{llll}
\hline Surface treatment & NMT1 (Direct bonding) & NMT2 (Direct bonding) & NMT1 (Adhesive bonding) \\
\hline Fracture energy $(\mathrm{J} / \mathrm{m})$ & $12.3 \pm 1.0$ & $11.6 \pm 1.1$ & $3.20 \pm 0.08$ \\
Lap shear strength $(\mathrm{MPa})$ & $44.2 \pm 1.2$ & $42.3 \pm 0.52$ & $10.8 \pm 0.22$ \\
Tensile strength $(\mathrm{MPa})$ & $43.0 \pm 2.5$ & $41.2 \pm 2.2$ & $18.5 \pm 1.2$ \\
\hline
\end{tabular}
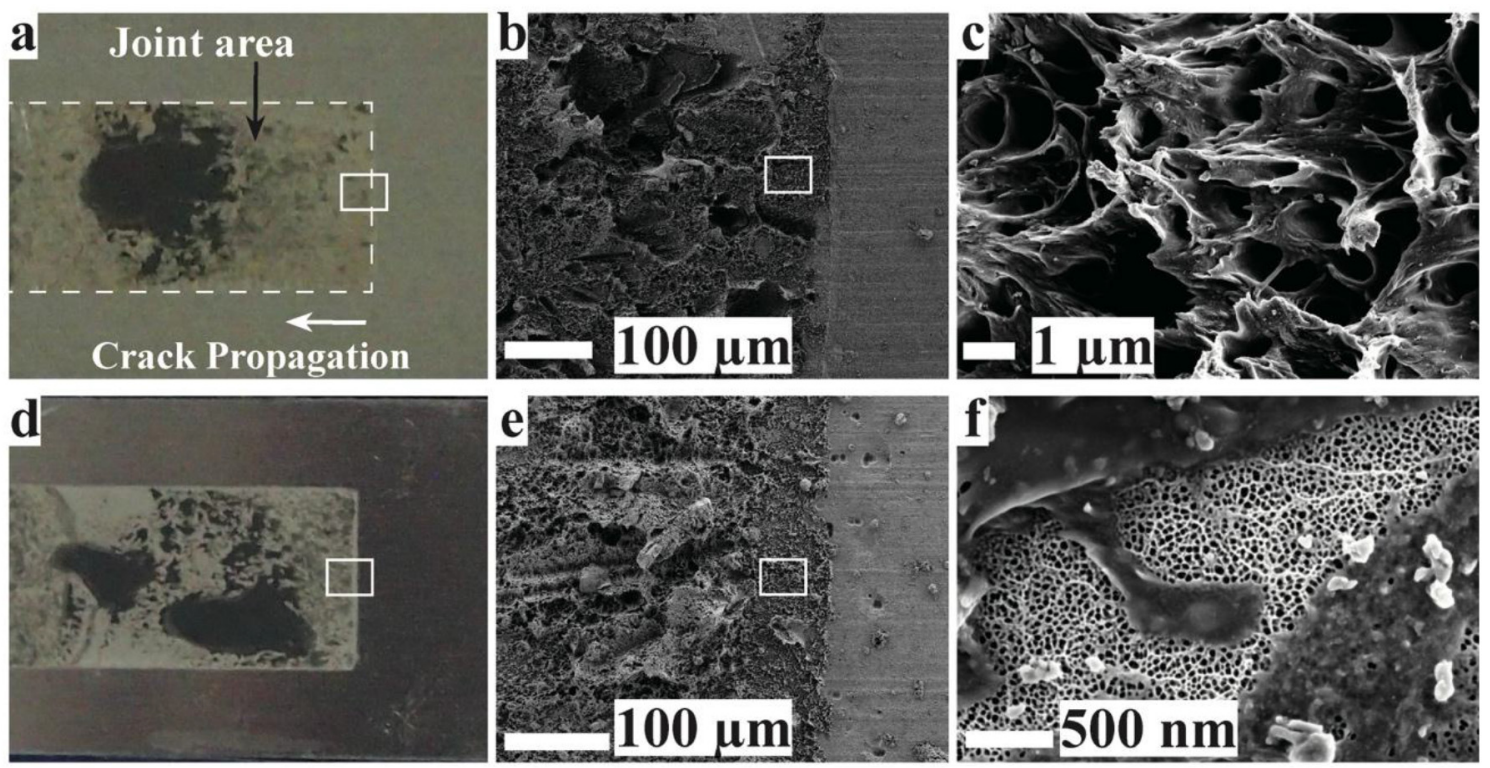

Fig. 14. Optical and SEM micrographs showing the fracture surfaces of Al5052 after fracture energy measurements. (a)-(c): NMT1 and (d)-(f): NMT2 surface treatments. (b) and (c) are SEM micrographs magnifying the regions indicated in (a) and (b), respectively. (e) and (f) are SEM micrographs magnifying the regions indicated in (d) and (e), respectively.
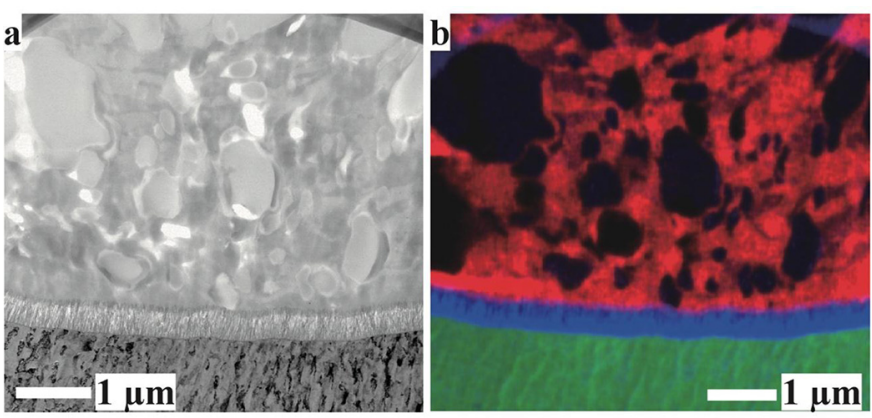

Fig. 15. (a) STEM-BF cross-sectional image and (b) STEMEDX mixed map of $\mathrm{S}$ (red), $\mathrm{O}$ (blue), and $\mathrm{Al}$ (green) of the fracture surface of $\mathrm{Al}$ after the fracture test. The surface treatment is NMT2.

The failure mechanism was investigated more in detail by cross-sectional images of the fracture surfaces by STEM. Figure 15a shows the STEM BF image of NMT2, exhibiting largely deformed PPS attached to the $\mathrm{Al}$ surface. Figure 15b is a mixed elemental map of S (red), O (blue) and $\mathrm{Al}$ (green), indicating that PPS is not pulled-off from the $\mathrm{Al}$ surface layer in this region. Figure 16 shows the NMT1 fracture surface near the crack tip, where Figure 16a and $\mathrm{b}$ are $\mathrm{a}$ STEM-HAADF image and STEM-EDX mixed
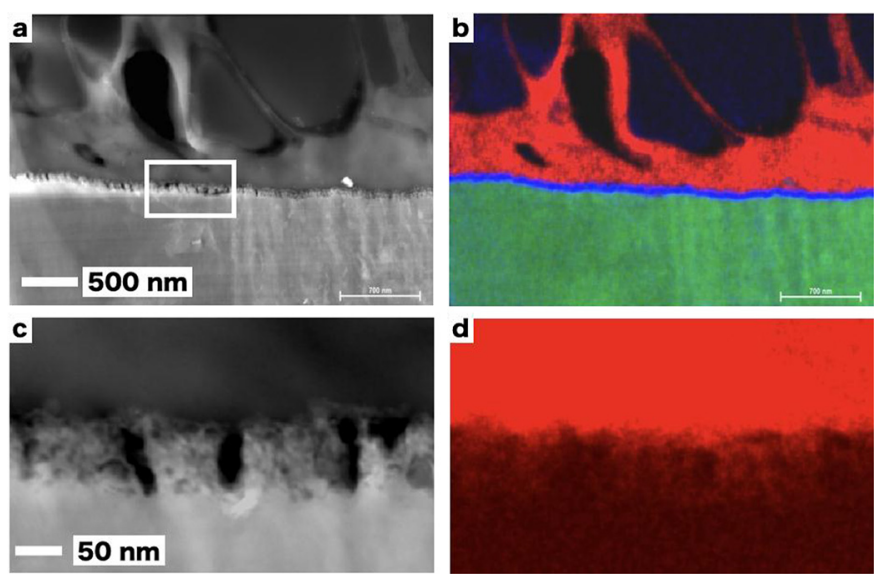

Fig. 16. (a) STEM-HAADF cross-sectional image and (b) STEM-EDX mixed map of S (red), O (blue), and Al (green) of the fracture surface of Al5052 after the fracture energy measurement. The surface treatment is NMT1. (c) Magnified image of the region indicated in (a), and (d) is STEM-EDX S map of (c) representing PPS distribution.

elemental map, respectively. This shows that the largely deformed PPS is attached onto the $\mathrm{Al}$ surface. Figure $16 \mathrm{c}$ is the magnified view of the interfacial region indicated in 
Figure 16a, which shows that the surface modified layer is partially damaged with voids. The STEM-EDX S map shown in Figure 16d indicates that PPS remains inside of the pores and is not pulled-off from the pores. This indicates that once PPS is penetrated into the pores of the surface treated by NMT1, it cannot be pulled-off from the interfacial layer, and instead of that, the interfacial layer is damaged to produce voids. This failure behavior is the reason for obtaining the higher joint strength than that of NMT2.

\section{Conclusions}

Plastic and metal can be directly bonded with remarkable strong joint strength via insert injection molding by creating surface porous structures with $30-50 \mathrm{~nm}$ sizes on metal surface. Molten polymers can be penetrated into the small pores in the short molding process, giving much higher joint strength than obtained by adhesive bonding in this work. We studied the bonding mechanism of $\mathrm{Al}$ and PPS with two different surface features of Al5052. Those two surfaces have different 3D connectivity in the modified surface layer. STEM-tomography technique allows us to see the inter-connectivity of the pores at the joint interfaces. One has three-dimensionally co-connected pores, while the other has isolated two-dimensional pores that are grown perpendicular to the metal substrate. With the 3D connected porous structure, the pores can be filled with polymers due to the ease of the replacement of air with polymer chains. We also found that the chemical reaction takes place during the joint process. The $\mathrm{Al}$ surface is in the meta-stable aluminum hydro-oxide state by the wet chemical treatment, and dehydration occurs during the bonding process, which convert aluminum hydro-oxide into more stable alumina. Both the $3 \mathrm{D}$ porous structure and the dehydration process might be essential for the polymer/metal strong joint interfaces.

A new test method for determination of the fracture resistivity of the joint interfaces was developed, and also the fast crack propagation and the strain concentration at the crack tip were successfully visualized by the ML technique with high-speed camera. No significant differences were given by the two interfacial structures, which mean that even though the penetration of PPS into the pores is incomplete, sufficient joint strength was achieved. It is anticipated that with the nano-sized porous metal structures, polymer chains and metal are strongly interacted. Different metal-plastic combinations and the application of the surface treatment technique employed in this work to adhesive bonding will be investigated in future.

This work was supported by JST-Mirai Program Grant Number JPMJMI18A2, Japan.

\section{References}

1. M.D. Banea, M. Rosioara, R.J.C. Carbas, L.F.M. da Silva, Multi-material adhesive joints for automotive industry, Compos. Part B 151 (2018) 71-77

2. S. Horiuchi, T. Hanada, T. Miyamae, T. Ymanaka, K. Osumi, N. Ando, M. Naritomi, Analysis of metal/plastic interfaces by energy-filtering transmission electron microscopy, J. Adhesion Soc. Jpn. 48 (2012) 322-330

3. M. Naritomi, N. Ando, M. Takahashi, M. Shiraishi, Composite material of aluminum alloy and resin and production method therefor, EP1459882A1.2004-09-22

4. ISO19095. Plastics-evaluation of the adhesion interface performance in plastic-metal assemblies, Part1-4, 2015

5. W. Dong, H. Hakukawa, N. Yamahira, Y. Li, S. Horiuchi, Mechanism of reactive compatibilization of PLLA/PVDF blends investigated by scanning transmission electron microscopy with EDX and EELS, ACS Appl. Polym. Mater. 1 (2019) 815-824

6. J. Franc, Electron tomography methods for three-dimensional visualizatin of structures in the cell, 2nd edn. Springer, Berlin, 2005

7. J.F. Watts, J. Wolstenholme, An introduction to surface analysis by XPS and AES, 2nd edn., Wiley, West Sussex, UK, 2020

8. N. Terasaki, Y. Fujio, Y. Sakata, S. Horiuchi, H. Akiyama, Visualization of crack propagation for assisting double cantilever beam test through mechanoluminescence, J. Adhesion 94 (2018) 867-879

9. N. Terasaki, Y. Fujio, S. Horiuchi, H. Akiyama, Mechanoluminescent studies of failure line on double cantilever beam (DCB) and tapered-DCB (TDCB) test with similar and dissimilar material joints, Int. J. Adhes. Adhes. 93 (2018) 40-46

10. L.H. Miligan, The mechanism of the dehydration of crystalline aluminum hydroxide and of the adsorption of water by the resulting alumina, J. Phys. Chem. 26 (1922) $247-255$

11. Z.D. Zivković, B. Dobovišek, Kinetics of aluminium hydroxide dehydration, J. Therm. Anal. 12 (1977) 207-215

12. S. Wu, Polymer Interface and Adhesion, Marcel Dekker, New York, 1982

13. ISO 13586. Plastics - Determination of fracture toughness (GIC and KIC) - Linear elastic fracture mechanics (LEFM) approach, 2018

Cite this article as: Shin Horiuchi, Nao Terasaki, Masami Itabashi, Evaluation of the properties of plastic-metal interfaces directly bonded via injection molding, Manufacturing Rev. 7, 11 (2020) 